\title{
Study of globular cluster M53: new variables, distance, metallicity $\star, \star \star$
}

\author{
I. Dékány and G. Kovács
}

Konkoly Observatory, PO Box 67, 1525 Budapest, Hungary

e-mail: dekany@konkoly.hu

Received 17 April 2009 / Accepted 27 August 2009

\section{ABSTRACT}

\begin{abstract}
Aims. We study the variable star content of the globular cluster M53 to compute the physical parameters of the constituting stars and the distance of the cluster.

Methods. Covering two adjacent seasons in 2007 and 2008, new photometric data are gathered for 3048 objects in the field of M53. By using the OIS (optimal image subtraction) method and subsequently TFA (trend filtering algorithm), we search for variables in the full sample by using discrete Fourier transformation and box-fitting least squares methods. We select variables based on the statistics related to these methods combined with visual inspection.

Results. We identified 12 new variables (2 RR Lyrae stars, 7 short periodic stars -3 of them are SX Phe stars - and 3 long-period variables). No eclipsing binaries were found in the present sample. Except for the 3 (hitherto unknown) Blazhko RR Lyrae (two RRab and an RRc) stars, no multiperiodic variables were found. We showed that after proper period shift, the PLC (period-luminositycolor) relation for the first overtone RR Lyrae sample tightly follows the one spanned by the fundamental stars. Furthermore, the slope is in agreement with that derived from other clusters. Based on the earlier Baade-Wesselink calibration of the PLC relations, the derived reddening-free distance modulus of M53 is $16.31 \pm 0.04 \mathrm{mag}$, corresponding to a distance modulus of 18.5 mag for the Large Magellanic Cloud. From the Fourier parameters of the RRab stars we obtained an average iron abundance of $-1.58 \pm 0.03$ (error of the mean). This is $\sim 0.5$ dex higher than the overall abundance of the giants as given in the literature and derived in this paper from the three-color photometry of giants. We suspect that the source of this discrepancy (observable also in other, low-metallicity clusters) is the lack of a sufficient number of low-metallicity objects in the calibrating sample of the Fourier method.
\end{abstract}

Key words. globular clusters: individual: M53 - methods: data analysis - stars: variables: general - stars: variables: RR Lyr stars: oscillations - stars: abundances

\section{Introduction}

Among the oldest and most metal-poor Galactic globular clusters, the outer halo cluster M53 (NGC 5024, $\alpha=13^{\mathrm{h}} 12^{\mathrm{m}} 55^{\mathrm{s}} \cdot 3$, $\left.\delta=+18^{\circ} 10^{\prime} 9^{\prime \prime}\right)$ is the second most abundant in variable stars after M15 (Clement et al. 2001, see also Marín-Franch et al. 2009 for current age determinations). The search for its RR Lyrae content dates back to Shapley (1920), and has yielded a total of 60 RR Lyrae stars. A thorough review of discoveries is given in Kopacki (2000), the only CCD time-series photometric study of M53 RR Lyrae stars prior to this work. This cluster is also very abundant in blue straggler stars (BSSs). Among the almost 200 BSSs, 8 were proven to be SX Phe variables (Jeon et al. 2003). Beccari et al. (2008a) showed that the BSSs follow a bimodal distribution, implying a binary rate of $10 \%$, but no eclipsing binaries have been found so far. As for the most extensive color-magnitude study of M53, we refer to Rey et al. (1998). The cluster is located at a very high Galactic latitude of $b=79^{\circ} .76$. Therefore, its field contamination and interstellar reddening is expected to be negligible. This is confirmed by Zinn (1985), reporting $E(B-V)=0.0$, and by Schlegel et al. (1998), who give

* Table 1 is only available in electronic form at http: //www . aanda. org

$\star \star$ Photometric data are only available in electronic form at the CDS via anonymous ftp to cdsarc.u-strasbg.fr (130.79.128.5) or via http://cdsweb.u-strasbg.fr/cgi-bin/qcat?]/A+A/507/ 803 a reddening value of $E(B-V)=0.02$. Despite its high variable content and its favorable position, no comprehensive wide-field CCD time-series photometric study has been published for this cluster. Among the two previous works of similar type, Kopacki (2000) observed only the central part of the cluster, while Jeon et al. (2003) limited themselves to the investigation of SX Phe stars. The present study, based on two-color photometry covering most of the cluster, intends to improve the time and spatial coverage of this cluster.

To exploit the entirety of our photometric data-base and detect as many variables as possible, we employ the widely used method of optimal image subtraction (OIS) developed by Alard \& Lupton (1998) and improved by Alard (2000), to obtain optimum relative light curves. Then, in a post-processing phase, we filter out temporal trends by using the trend filtering algorithm (TFA) of Kovács et al. (2005). This latter method has been extensively used during the past several years by the HATNet Project ${ }^{1}$ in the search for transiting extrasolar planets and also employed successfully in general variable searches (Kovács \& Bakos 2008; Szulágyi et al. 2009). The main purpose of this paper is to search for variables in the full dataset and not limit the investigation to the well-known regions of variability (i.e., the RR Lyrae strip, the blue straggler and the upper red giant branch regions of the Hertzspring-Russell diagram). With the aid of the reduction and

\footnotetext{
1 Hungarian-made Automated Telescope Network, see http: //cfa-www . harvard. edu/ gbakos/HAT
} 
post-processing methods mentioned above, we can increase the photometric precision and attempt to reach the detection limit set by the photon statistics.

In Sect. 2 we give details on the observations and data processing, and show examples of the detections resulting from the methods applied. The next three sections (Sects. 3-5) are devoted to a more detailed analysis of the specific types of variables, most importantly, to that of the RR Lyrae stars. Our attention will be focused mostly on the period-luminosity-color relation and the metallicity issue. A brief discussion and conclusions are given in Sect. 6.

\section{Observations, data reduction, method of analysis}

\subsection{Observations and data reduction}

We performed time-series photometric observations of M53 through $V$ and $I$ filters of the Johnson and Kron-Cousins system, respectively, with the $60 / 90 / 180 \mathrm{~cm}$ Schmidt Camera of the Konkoly Observatory located at Piszkéstetô. The telescope is equipped with a Photometrics AT 200 type CCD of 9- $\mu$ pixels in a $1536 \times 1024$ array. The point spread function (PSF) is slightly undersampled with a scale of $1.026 \mathrm{arcsec} / \mathrm{pixel}$. The observations were carried out between March 25, 2007 and May 28, 2008 in two seasons, on a total number of 26 nights. The observations have a full time span of 430 days. Exposure times varied between 550 and $750 \mathrm{~s}$ in $V$ and 350 and $540 \mathrm{~s}$ in $I$ depending on atmospheric conditions. A total of 340 and 230 frames have been collected in the $V$ and $I$ bands, respectively. While for the time series analysis we use only the $V$-band data, for standard magnitude transformations, variable star classification, and for deriving cluster distance and metallicity, we also utilize the I-band data.

The processing of the images (bias, dark, and flat-field corrections) and the alignment of all frames into a common pixel reference system were performed by standard $\mathrm{IRAF}^{2}$ packages. The joint field of view of all observations was somewhat truncated compared to the full detector area for technical reasons. Only this $13.5 \times 14.5$ common area (still covering a fairly large part of the cluster by spanning $\sim 0.7$ tidal radius, see the cata$\log$ of Harris 1996, revised in February 2003), centered approximately on the cluster core, underwent further reduction.

We calibrated the astrometric transformations between pixels and celestial coordinates by using 30 bright stars from the second edition of the Guide Star Catalog (version 2.3.2, Lasker et al. 2008) uniformly distributed around the cluster center and located sufficiently well outside the cluster core. The standard deviations in the residuals of the coordinate mapping were $0 .{ }^{\prime} 148$ and $0 . ' 163$ in right ascension and declination, respectively.

In order to deal with heavy crowding and to probe the regions close to the center of the cluster, we applied the OIS method of Alard (2000). For this method one has to prepare an accurate reference frame (RF) in each band which will be subtracted from all other frames after the appropriate scaling of the PSF of the stellar objects. The success of the method is highly sensitive to the quality of the RFs, therefore we carefully selected the best images with the lowest background, best seeing, and most regular PSFs and stacked them into one single image in order to increase their signal-to-noise ratio. Before the subtraction from each frame, the RF was convolved with a kernel function, which

\footnotetext{
2 IRAF is distributed by the National Optical Astronomy Observatory, which is operated by the Association of Universities for Research in Astronomy, Inc., under cooperative agreement with the National Science Foundation.
}

was determined by using the representative PSFs of 225 stars uniformly distributed on the image. The kernel functions were allowed to have second order spatial variations to account for a varying PSF across the image. The convolved RF, being the best approximation of the processed frame in a least-squares sense, was subtracted from the processed frame. The method yielded a set of residual images consisting of, in principle (but not in practice), the true light variations plus noise. For all procedures of image convolution and subtraction we used the ISIS 2.2 package of Alard (2000).

We applied the DAOPHOT PSF-fitting software package (Stetson 1987) on the RFs to identify stellar objects and to obtain their instrumental magnitudes. We found 3048 stars above the $10 \sigma$ level. Linear transformation equations of these instrumental magnitudes into standard ones were established by using 110 photometric standard stars of Stetson (2000) around the cluster center.

We measured differential flux variations in the residual frames by aperture photometry at all stellar positions obtained from the RFs, using a custom IRAF routine. The sizes of the apertures were variable in order to conform to the FWHMs of the PSFs on the original frames, changing in time due to seeing variations. To determine whether some faint variables might have remained undetected on the RFs due to crowding but with light variations emerging on the residuals, we also employed the detection algorithms of ISIS 2.2 in two ways. First, all sources of significant variability were checked for the case of light variation coming from a yet undetected faint star falling within the same aperture. In these cases we performed its photometry using a new, accurately recentered aperture. Secondly, we checked if there were any remaining faint variables that had neither been detected on the RFs nor fell within any aperture (we did not find any additional variables by this latter test). All light curves obtained in this way, expressed in linear flux units, were then further processed with the aid of the trend filtering algorithm (TFA, see Sect. 2.2 for further details).

As the last step of the reduction, to convert differential fluxes of an object into magnitudes, one has to know the flux $f_{\mathrm{RF}}$ of the source on the RF in each band which has been subtracted from each processed frame (see e.g., Wozniak 2000). This additional information sets the zero point of the logarithmic transformation

\section{$\Delta m_{i}=-2.5 \log \left(f_{\mathrm{RF}}+\Delta f_{i}\right)$}

between the $\Delta m_{i}$ differential magnitude and $\Delta f_{i}$ differential flux of the object (derived by the OIS method) on the $i$ th frame. The $f_{\mathrm{RF}}$ value has been measured by PSF photometry for each object as described above. Clearly, a flux-to-magnitude transformation with an imprecise $f_{\mathrm{RF}}$ value yields an incorrect amplitude of the light variation and can significantly alter the magnitude averages as well, but its actual effect on these errors is specific to each object and depends on the brightness and the shape of the light variation. Therefore, the flux curves of variable stars were transformed into magnitudes only if their (already standardized) photometry on the RFs were reliable enough, which has been decided based on the formal errors of the PSF photometry. Generally, objects were transformed into magnitudes if the formal error of their $(V-I)_{\mathrm{RF}}$ was below $0.03 \mathrm{mag}$ (see Sect. 3.2 for details on this cut in the case of RR Lyrae stars) ${ }^{3}$.

\footnotetext{
3 Photometric time-series of the variables detected in this work and the Fourier decompositions of the RR Lyrae stars with magnitudetransformed light curves in $V$ color are available online at CDS.
} 


\subsection{Post-processing with TFA}

The time-series data-base obtained above is subjected to a subsequent post-processing phase, when we filter out various trends/systematics due to, e.g., imperfect reduction, lack of correction to position- and time-dependent extinction, anomalies in the convolution procedure prior to image subtraction, etc. The method is described in detail by Kovács et al. (2005) and also summarized recently by Bakos et al. (2009) and Szulágyi et al. (2009). Here we only briefly note that the method is based on the idea of correcting elements of the systematic variation in the target time series by using the light curves of many other objects, available in the CCD frame. In the course of the signal search, we have no information on the temporal content of the time-series. Therefore, we assume that the target is trend- and noise-dominated. If it is the case, then the method suppresses the trend and allows the signal to appear in some time-frequency transformation of the detrended signal. Here, at the expense of the suppression of the systematics, we also deform the true signal at some level. This effect is removed in the second step of filtering, when we reconstruct the signal by using a full time-series model that includes both the systematics and the signal with the period obtained in the first step. An extension of the method to multiperiodic signals is given in Kovács \& Bakos (2008). In this method (also adopted here) one fits the TFA template and the signal (represented by a Fourier series) simultaneously, thereby avoiding iteration by incomplete time-series model representations.

Special attention is to be paid here to the effects related to the number of data points. In the ideal case, the number of TFA templates necessary to handle all systematics is considerably lower than the number of data points constituting the time-series. Unfortunately, we do not have a good method to select a "representative" small set of templates, therefore we use a large set that includes both "useful" and "useless" (e.g., pure noise) template time-series. Attempts have been made to determine an optimum number of templates but it seems that it is hard to settle at a value lower than a few hundred (Szulágyi et al. 2009). Since in the present case we have only 340 data points per time-series, we have to limit the number of templates considerably (i.e., about half of the number of the data points) to avoid overfitting and a sudden increase of the false alarm probability (FAP). This latter effect is thoroughly tested in each interesting case by using multiple template runs, which are capable of reducing FAP by several factors (Kovács \& Bakos 2007). To avoid significant overfitting, we settled on the lowest TFA template numbers that yielded the most significant detection together with the lowest unbiased scatter around the signal. This optimum template number varied between 60 and 140, the latter being an upper limit set by us due to the low number of available data points.

In general, we selected the TFA template sets from the $\sim 1000$ brightest stars in the sample. The faintest stars in the template sets did not exceed $\sim 18$ mag in $V$ band. The templates cover the frame in a quasi-uniform manner (see Kovács et al. 2005 , for more details on the distribution of templates).

The significance of the Fourier or BLS components (see Kovács et al. 2002) was deduced by checking the signal-to-noise ratio (SNR) of the frequency spectra (the ratio of the amplitude of the highest peak to the standard deviation of the spectrum). Statistical tests (similar to the ones performed in our earlier papers, e.g., in Nagy \& Kovács 2006) have led to the conclusion that for Gaussian signals with $S N R>6.7$, the FAP due to uncorrelated random noise is less than $1 \%$. Considering that the processed signals always have some colored noise (either

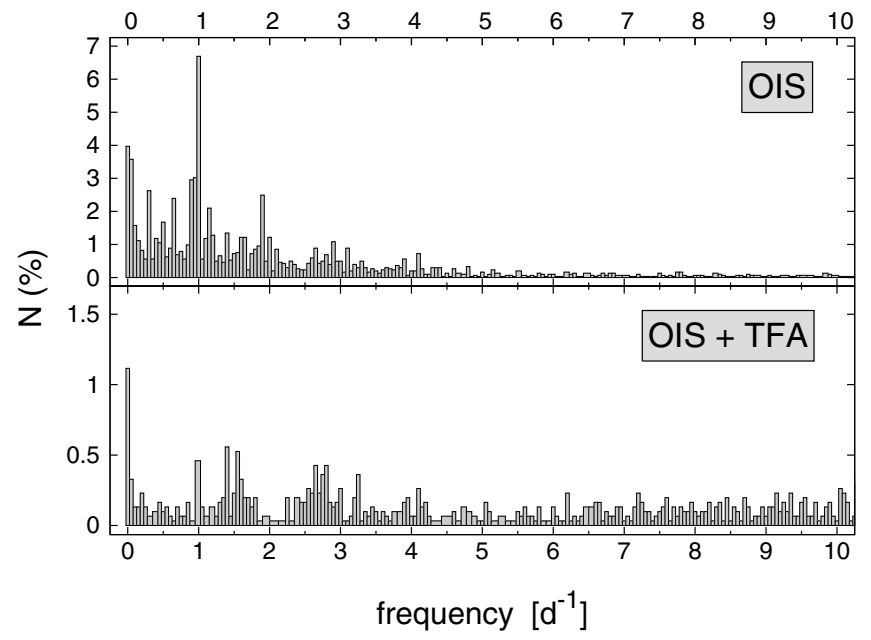

Fig. 1. Histogram of the peak frequencies of the Fourier (DFT) spectra for the 3048 stars analyzed. Upper panel shows the frequency distribution of data obtained by aperture photometry on the residual frames of the OIS method. Lower panel shows the effect of TFA on these data.

physical or instrumental) we selected variables well above this SNR value (usually with an SNR exceeding 8).

In demonstrating the signal detection, first we compare the distributions of the peak frequency components obtained by OIS and by the subsequent application of TFA on the OIS time series. Figure 1 shows that the OIS data already are reasonably free of periodic systematics. When compared with earlier similar diagrams derived from data of aperture photometry (e.g., Kovács et al. 2005; Szulágyi et al. 2009), we see that the current data are much less dominated by the customary $1 \mathrm{~d}^{-1}$ systematics. We see in the bottom panel that TFA has successfully filtered out the daily trends. In the final dataset, the frequency distribution becomes nearly flat, with a slight surplus due to the RR Lyrae stars and the long-term irregular light variations of red giants.

In checking the effect of TFA in more detail, we plotted the DFT (Discrete Fourier Transformation) spectra and the folded light curves for the OIS and OIS+TFA data in the case of an RRc (V71, Fig. 2) and an SX Phe star (V78, Fig. 3). The effect of the signal reconstruction is large in both cases. The true variability of V71 would have been significantly more troublesome to decipher from the forest of high peaks in the original (OIS) data than in the clean, single-component spectrum of the TFAfiltered time-series. For V78 the situation is better because of the high frequency of the pulsation. Nevertheless, even in this case, the highest peak is near $1 \mathrm{~d}^{-1}$ (and its aliases), therefore, an automatic search that only looks for the highest peak in the spectra would not find this variable in the original (OIS) data. The improvement in the quality of the folded light curve derived from the reconstructed data is also substantial. However, the small scatter seen is somewhat biased, because of the large number of TFA templates used. The effect of the bias in the residual scatter is common to all data regressions. The fit introduces correlation among the original array elements that leads to less observed scatter as expected if only the deterministic (model-predicted) part of the signal would have been fitted. In general, when leastsquares fitting a data array of $N$ data points with $N_{\mathrm{p}}$ parameters (larger than exact model representation required) the residual rms will be $\sqrt{\left(N-N_{\mathrm{p}}\right) / N}$-times the value expected from the noise level in the original data (see also Szulágyi et al. 2009 for further details on this subject). For this reason, the unbiased standard deviations of the reconstructed light curves are $10 \%$ 


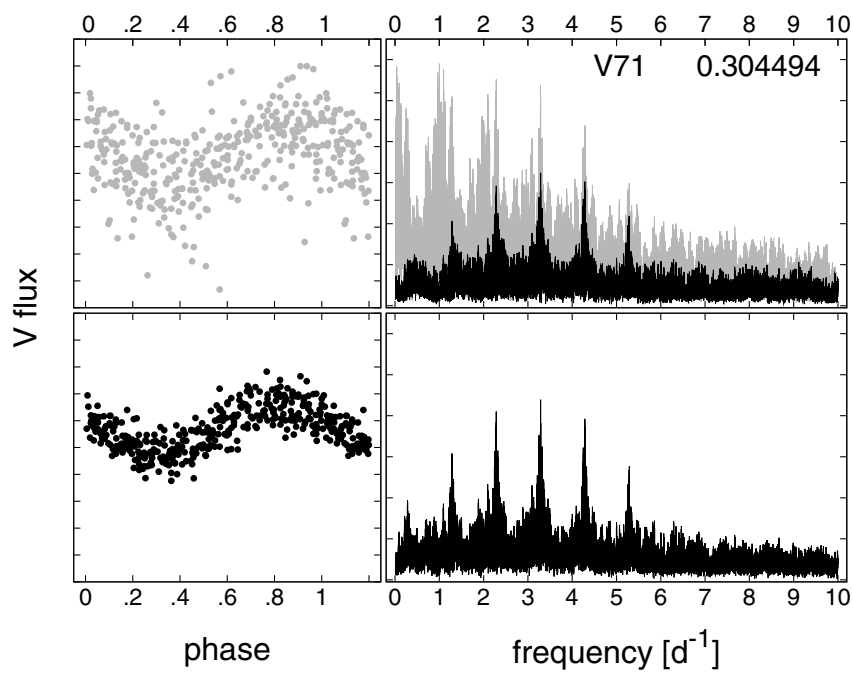

Fig. 2. Detection of the RRc variable V71. Top left: folded light curve (with the period detected by TFA) of the OIS data; top right: DFT of the OIS data (light shade) and that of the TFAd data (dark shade); bottom left: folded light curve of the TFA-reconstructed data; bottom right: DFT of the TFA-reconstructed data. Star identification name and period (in days) are shown in the upper right corner. See note in text on the scatter of the TFA-filtered light curve.

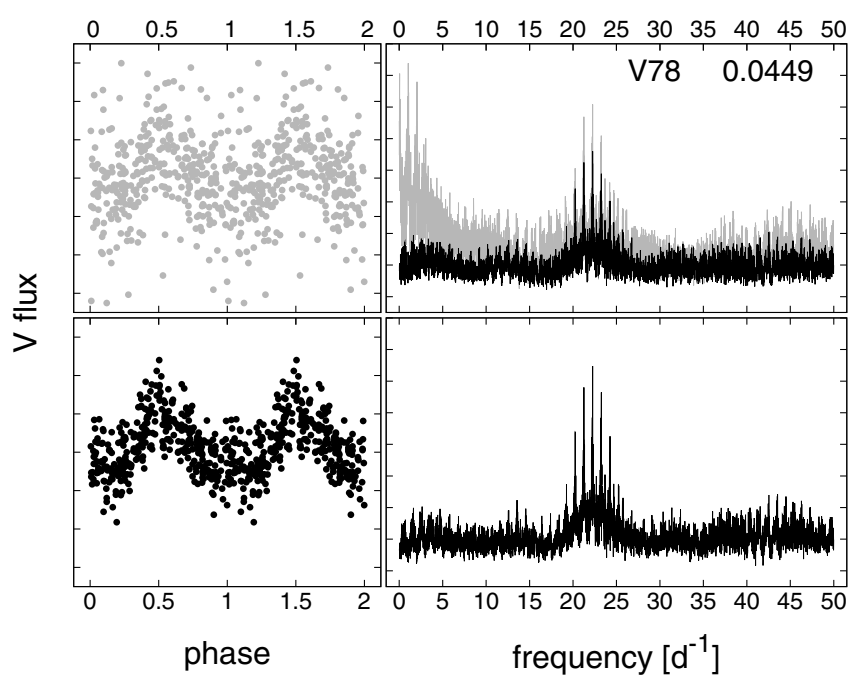

Fig. 3. Detection of the SX Phe star V78. Notation is the same as in Fig. 2.

and $30 \%$ higher due to the 60 and 140 templates used for V71 and V78, respectively. The different template numbers resulted from the multi-template tests mentioned earlier in this section, in the description of the method of analysis.

In a final example, in Fig. 4 we show the signal reconstruction capability of TFA. We used an 8th order Fourier series with 144 TFA templates in the reconstruction (see Kovács \& Bakos 2008). Inspection of the DFT spectrum of the OIS light curve of this star shows that it is not influenced by periodic systematics of type integer $\mathrm{d}^{-1}$. Therefore, the improvement introduced by TFA filters out some transient signals. We found transient systematics also in other variables, similarly to the ones reported by Szulágyi et al. (2009). The unbiased standard deviation of the residuals around the best-fitting Fourier-sum dropped by $14 \%$, which is a good improvement over the original data.

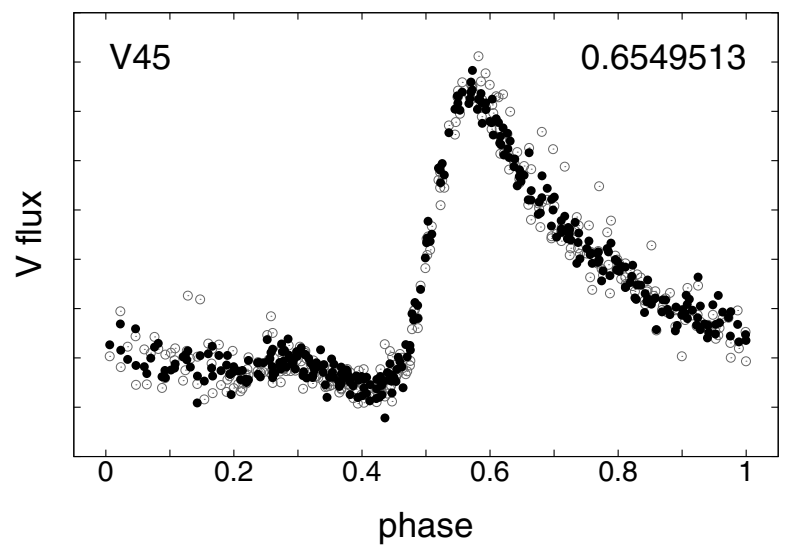

Fig. 4. Signal reconstruction in the case of the RRab star V45. Open circles denote the OIS fluxes, filled ones show the TFA-processed values. See note in text on the scatter of the filtered light curve.

\section{RR Lyrae stars}

Our prime interest in this paper is the study of the RR Lyrae stars, since they are present in large numbers in the cluster and they have fairly well established theoretical and observational understanding (e.g., variable and mode identification, computation of the physical parameters from observed ones, etc.). We focus on the distance and on the metallicity (i.e., $[\mathrm{Fe} / \mathrm{H}])$ as the two most important parameters derivable from the available empirical relations.

\subsection{General description}

We identified 54 RR Lyrae stars in the cluster. Except for the two RRc stars V71 and V72, all others were known previously (see the Catalog of Variable Stars in Globular Clusters, hereafter CVSGC, Clement et al. 2001). We note that the variability status of V34, which had been questioned previously by van den Hoven van Genderen (1947) was clearly confirmed by our data. Due to the application of OIS and TFA, many of the variables ended up with light curves suitable for further analysis. We give the full list of variables together with their basic observed parameters in Table 1. Please note the very high SNR attached to each detection even for the variables near the center of the cluster.

The accuracy of the periods of the RR Lyrae stars was estimated in the following way. First we computed the Fourier decompositions from the TFA-reconstructed light curves. From these decompositions, synthetic light curves were generated and Gaussian noise was added with standard deviations obtained from the unbiased estimates of the residual scatter between the synthetic light curves and the TFA reconstructions. The generated light curves were subjected to the standard Fourier frequency analysis as if they were real observed light curves. The periods obtained from each realization were stored and after completing 100 independent simulations for each object, the standard deviations of those period values were computed. The errors shown in Table 1 are these standard deviations.

The periods obtained in this study are generally in good agreement with previously determined values, except for the three RRc stars V44, V47, and V55, for which the previous values are various aliases of the true periods. In the case of other first overtone stars, the agreement between our and earlier published values are better than $0.0004 \mathrm{~d}^{-1}$ with a typical difference of about $0.00003 \mathrm{~d}^{-1}$. For RRab stars, deviations are 


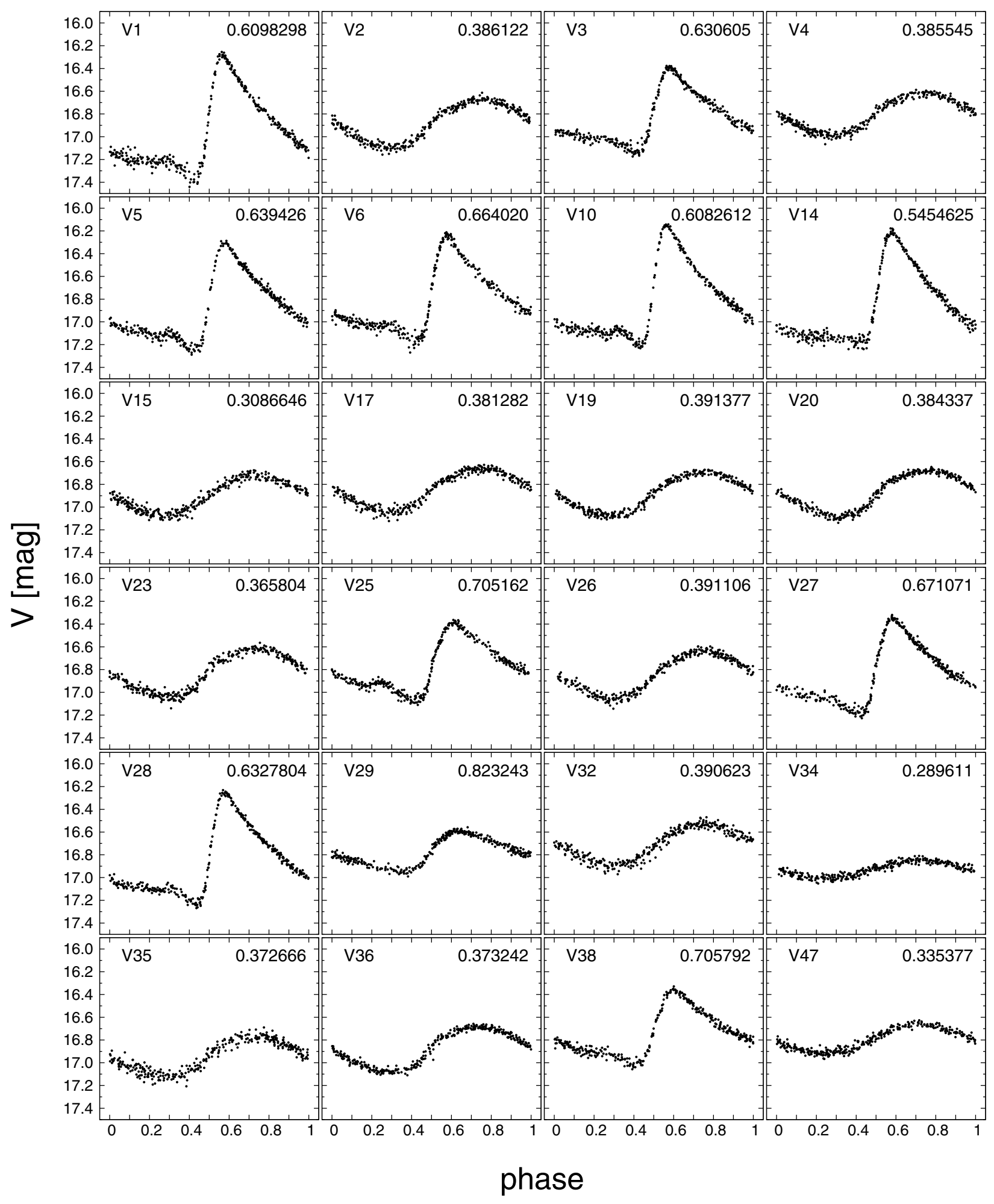

Fig. 5. Standard $V$ light curves of the 24 RR Lyrae stars for which the flux-to-magnitude conversion was performed. CVSGC numbers and periods in days are given in each case (see Table 1 for additional data). All light curves were reconstructed by TFA.

generally below $0.00002 \mathrm{~d}^{-1}$, with a few higher values not exceeding $0.00012 \mathrm{~d}^{-1}$.

Figures 5 and 6 show the TFA-reconstructed and folded light curves. Standardizing the photometry for the highly crowded 30 objects shown in Fig. 6 was not reliable enough, therefore, we left the light curves in relative flux units. Unfortunately, these objects can only be partially utilized here due to the lack of empirical relations derived from fluxes and the absence of existing photometry with a higher resolution CCD (exceptions are V7, $\mathrm{V} 8, \mathrm{~V} 9, \mathrm{~V} 33$, and V37 that have good-quality light curves in $V$ magnitude from Kopacki 2000). We note that among the previously known RR Lyrae stars missing from our sample, V52 and 


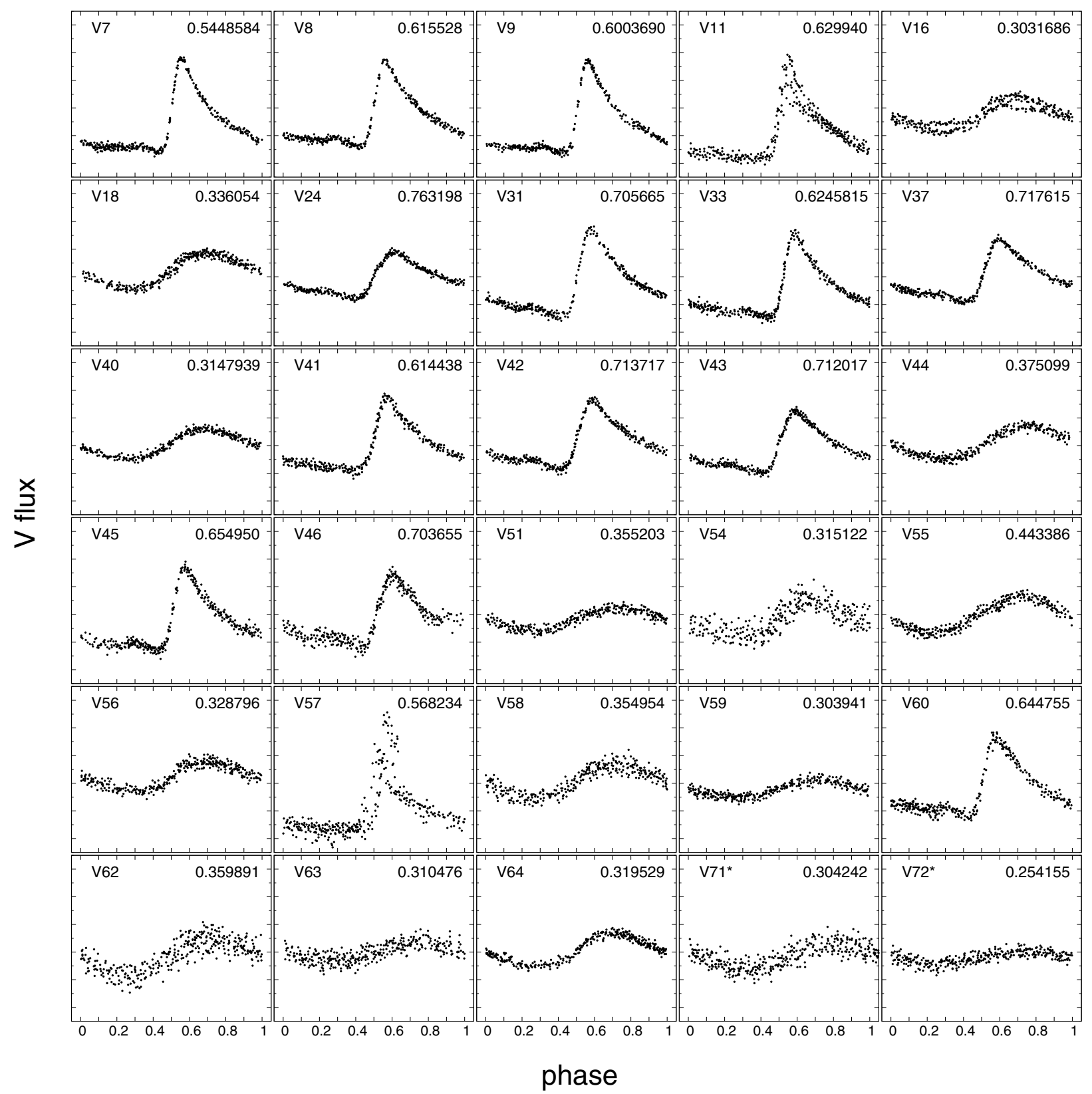

Fig. 6. Light curves expressed in arbitrary differential flux units of the $30 \mathrm{RR}$ Lyrae stars that were not transformed into magnitude values due to the large zero-point errors associated with crowding. All plots are shown on the same ordinate scale. CVSGC numbers and periods in days are given in each case (see Table 1 for additional data). All light curves were reconstructed by TFA.

V53 were merged, V61 was merged with the long period variable V49, and the rest (V12, V13, V21, V30, and V48) were outside the field of view.

\subsubsection{Blazhko-stars}

We investigated possible multiperiodicity by performing successive prewhitening on all 3048 objects, including RR Lyrae stars. Surprisingly, from the whole sample we found only the three Blazhko stars given in Tables 1 and 2, in spite of the relatively large scatter visible in several stars (see Fig. 6). We note that the detection of the Blazhko behavior in the three stars is firm. The modulation is clearly observable as side-lobe frequencies with uniform $f_{\mathrm{m}}$ frequency separations after prewhitening the main frequency and its harmonics. For V11, significant side lobe frequencies could be identified for up to the 7 th harmonic of $f_{0}$ at $n f_{0}+f_{\mathrm{m}}$, except for $n=2$, where we found $2 f_{0}-f_{\mathrm{m}}$. In the case of the RRc star V16 we found $f_{0}+f_{\mathrm{m}}$ and $2 f_{0}+f_{\mathrm{m}}$. The phase modulations in both stars are small, unlike that of the third Blazhko-star, V57, whose modulation is very large both in phase and in amplitude. We detected $f_{0}+f_{\mathrm{m}}$ and $2 f_{0}+f_{\mathrm{m}}$ in the case of V57. The light curves of the Blazhko stars folded with the pulsation periods are shown in Fig. 6. The light curves were reconstructed by simultaneously fitting the template and the full modulated signal (see Sect. 2.2).

\subsection{PLC relation}

The tightest empirical relations existing for RR Lyrae stars are the period-infrared $K$ magnitude $\left(\mathrm{PL}_{K}\right)$ (Longmore et al. 1990) 
Table 2. Modulation properties of the Blazhko-stars.

\begin{tabular}{clccc}
\hline \hline ID & Type & $f_{\mathrm{m}}\left[\mathrm{d}^{-1}\right]$ & $P_{\mathrm{m}}[\mathrm{d}]$ & $R_{\mathrm{m}, 1}$ \\
\hline V11 & RRab & 0.028 & 35 & 0.21 \\
V16 & RRc & 0.017 & 58 & 0.41 \\
V57 & RRab & 0.020 & 52 & 0.25 \\
\hline
\end{tabular}

Note: $R_{\mathrm{m}, 1}$ is the ratio of the amplitude of the largest modulation peak in the DFT spectrum and the amplitude of the $f_{0}$ pulsation component (see Table 1), in $V$ flux.

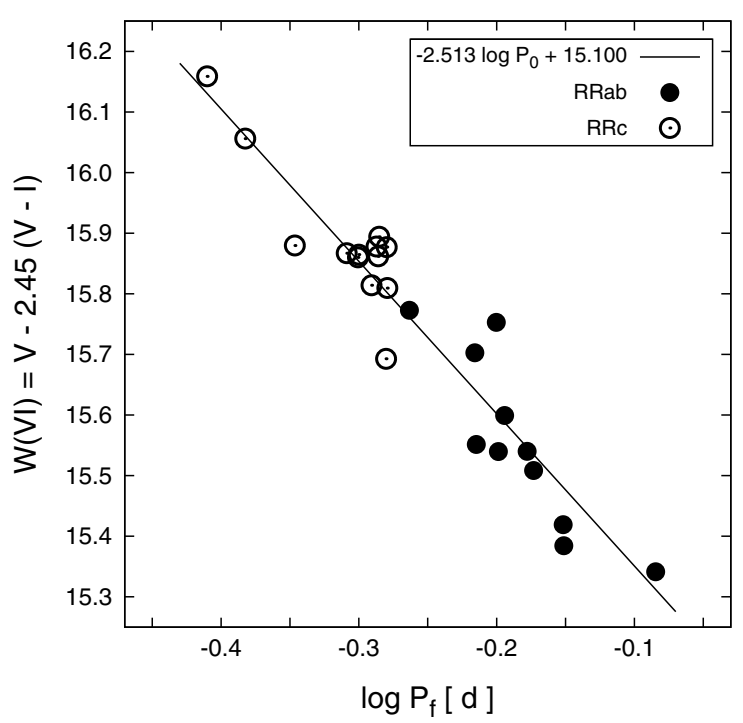

Fig. 7. PLC relation for 24 RR Lyrae stars in M53. RRc periods were shifted by adding 0.128 to their $\log P_{1}$. Continuous line shows the zeropoint fitted regression line with the slope given in KW01.

and the various period-luminosity-color (PLC) relations (see Kovács \& Walker 2001 [hereafter KW01]; Cortés \& Catelan 2008; Cáceres \& Catelan 2008). In addition to the theoretical interest in these relations (Bono et al. 2001; Di Criscienzo et al. 2004), they also have important practical utilization as a simple and accurate way to determine distances. Furthermore, it is also an interesting question as to whether RRc stars (after a proper period shift) fit the empirical PLC relation spanned by the RRab stars. Since M53 contains a considerable number of RRc stars, we can address this question with reasonable confidence here (see also Cassisi et al. 2004 concerning the same problem in the case of the variables in M3). To decrease the number of parameters fitted, we use the slope of the PLC relation (i.e., $\log P-$ Wesenheit index relation ${ }^{4}$ ) derived by KW01 from globular cluster RRab variables, for the $V$ and $I$ magnitudes. (We note that this slope is in agreement with the one derived from the large - but considerably noisier - sample of LMC RRab stars by Soszyński et al. 2003).

Figure 7 shows the derived joint PLC relation. The periods of the RRc stars have been increased by $-\log \left(P_{1} / P_{0}\right)=$ 0.128 to take proper account of the overall period difference between the RRab and RRc stars. The above shift corresponds to $P_{1} / P_{0}=0.745$, which is the average expected period ratio for

\footnotetext{
${ }^{4}$ By construction, the Wesenheit index is reddening-free in the case of the "standard" interstellar reddening law (e.g., Cardelli et al. 1989). Interestingly, the best-fitting PLC relation with free-floating color coefficients yields values very close to those predicted by the standard extinction law (see Kovács \& Jurcsik 1997; Udalski et al. 1999).
}

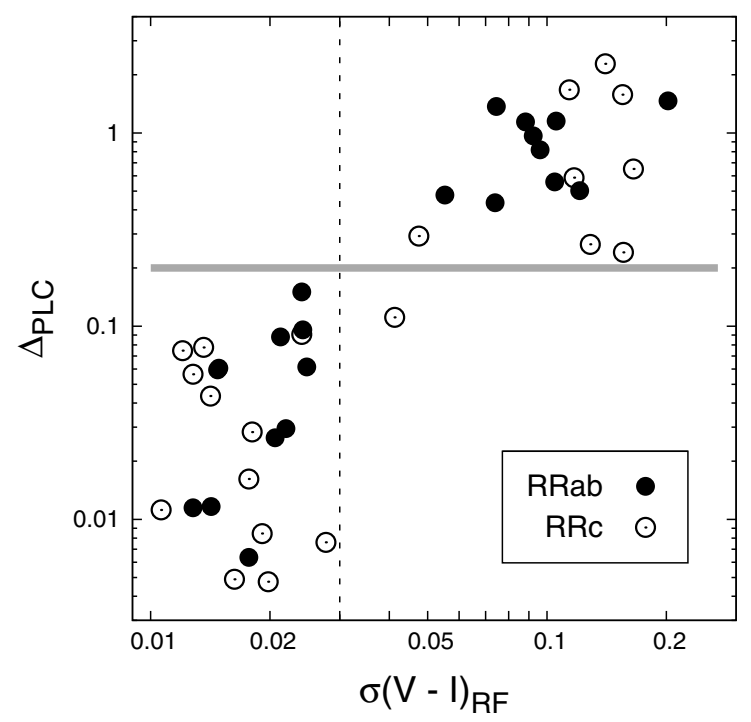

Fig. 8. Distance from the empirical PLC relation versus the error of the color index computed from the formal errors of the object images on the reference frames. Gray horizontal line shows the near $3 \sigma$ limit of the scatter around the regression line of the empirical relation of KW01. The dashed vertical line indicates the cutoff error for the color index as explained in the text.

the first overtone and fundamental mode periods for RR Lyrae stars (see, e.g., Cox et al. 1983). From the 54 RR Lyrae stars with available $V, I$ photometry we omitted 30 stars due to their outlier status in the PLC relation (the stars without magnitude averages in Table 1). We investigated the question of whether the omission of these stars can be justified based on the behavior of some other parameters derived during the reduction of the data. The first parameter to be utilized for this purpose could be the scatter of the light curve. But while crowding introduces large errors in the zero points of the flux-to-magnitude conversion, this may not necessarily lead to an increase of the scatter of the light curve due to the effectiveness of the OIS method (compare variable positions in Table 1 and light curve scatters in Fig. 6). Therefore, we resorted to the inspection of another quantity that might correlate with the zero point errors of the light curves. This quantity is the formal error of the RF magnitude from the standard method of PSF photometry. For a considerable number of stars, the discrepant position in the PLC relation was coupled with an obviously discrepant amplitude of the light curve. This is evidently introduced by the incorrect conversion of their fluxes into magnitudes (see Sect. 2.1) due to the inaccuracy of their RF photometry. However, it is important to justify whether the same photometric error (and not, e.g., non-cluster membership) can account for the outlier status of all the omitted stars. The correlation of the formal errors of $(V-I)_{\mathrm{RF}}$ with the (vertical) distance from the best-fitting PLC relation is exhibited in Fig. 8. Indeed, all omitted stars also have larger photometric formal errors. Therefore, we think that the data clipping used in cleaning the PLC relation is justified. On the other hand, the "good quality stars" (defined as with a formal $(V-I)_{\mathrm{RF}}$ error of $\lesssim 0.03$ mag, located below the gray line in Fig. 8) show various levels of agreement with the ridge line of the PLC, ranging from a perfect match to near $3 \sigma$ difference. This indicates that formal image errors can serve as a guide only when strong discrepancies are observed in applications involving photometric data derived from the lower quality images. The final standard deviation of 
the fit is $0.063 \mathrm{mag}$, slightly worse than the one derived from several clusters by KW01.

For the zero-point calibration of this PLC relation, we relied on the Baade-Wesselink (BW) analysis of Kovács (2003). From the 21 BW stars with $V-I$ color indices, we omitted AV Peg, because of its strongly discrepant position in the PLC relation. Six additional stars (X Ari, SW Dra, SS Leo, V445 Oph, BB Pup and $\mathrm{W}$ Tuc) could also be omitted but this does not lead to a significant change in the zero point of the BW PLC relation $(-1.212 \pm 0.037$ vs. $-1.182 \pm 0.026$ with the six stars omitted).

By fixing the slope of the $\log P_{0}$ term as given by KW01 for $W(V, I)=V-2.45(V-I)$, for the $24 \mathrm{RRab}$ and RRc stars of M53 we get

$W(V, I)=-2.513 \log P_{0}+(15.100 \pm 0.013)$,

where the error corresponds to the $1 \sigma$ formal statistical error. Similarly, for the $20 \mathrm{BW}$ stars we get

$W(V, I)_{\mathrm{BW}}=-2.513 \log P_{0}-(1.212 \pm 0.037)$.

Simple subtraction of the zero points of the above two equations yields $16.312 \pm 0.039$ for the dereddened distance modulus of the cluster. We note that this value is compatible with an LMC distance modulus of 18.5 mag (Kovács 2003, see also the recent summary of di Benedetto 2008). Additional notes on the distance of the cluster are given in Sect. 3.3 in relation to the metal content and various $M_{V}-[\mathrm{Fe} / \mathrm{H}]$ relations.

\section{3. $[\mathrm{Fe} / \mathrm{H}]$ distribution}

The application of the empirical formula for the computation of the iron abundance of RRab stars (Jurcsik \& Kovács 1996, hereafter JK96) has been proven to be quite successful in the past (Jurcsik \& Kovács 1996 [comparison for several GCs]; Székely et al. 2007 [NGC 362]). However, there is a growing concern about the accuracy of this formula at the low-metallicity end (e.g., Nemec 2004 [NGC 5053]; Garcia Lugo et al. 2007 [M15]; see however Kinemuchi et al. 2008 [Draco RRab vs. giant metallicities]). Since M53 is also a low-metallicity cluster, and the available light curves are of high quality for computing precise Fourier decompositions, here we can examine this problem in more detail.

In tracing back the metallicity estimates derived for this cluster, we found the works of Zinn \& West (1984, hereafter ZW84) and Suntzeff et al. (1988, hereafter S88). The metallicities presented in these papers are both based on calibrated spectral indices (similar to the $\Delta S$ index of Preston on which the JK96 formula is calibrated). The two studies, based on the analyses of several giant stars, yield very similar metallicities for M53 $\left(-2.04 \pm 0.08\right.$ by ZW84 based on the $Q_{39}$ parameter, and $-2.09 \pm 0.09$ and $-2.20 \pm 0.33$ by $\mathrm{S} 88$ based on the $m_{\mathrm{HK}}$ and $m_{\mathrm{Mg}}$ indices, respectively). A high-dispersion detailed abundance analysis on a single giant was made by Pilachowski et al. (1983). They obtained a value of -1.9 . Additional early $[\mathrm{Fe} / \mathrm{H}]$ estimates are also listed in their paper. Most of the values indicate a metallicity lower than -1.8 .

To further confirm the low metallicity of the giants of M53, we employed the photometric method of Kovács (2008) (see also Dékány et al. 2008). The method is based on the well-known observation that the $B-V$ color index depends fairly sensitively on the gravity and more importantly on the metallicity. Because the most relevant parameters determining the theoretical values of the broad-band colors are the effective temperature, gravity and overall metal content $([\mathrm{M} / \mathrm{H}]$, identical to $[\mathrm{Fe} / \mathrm{H}]$, assuming

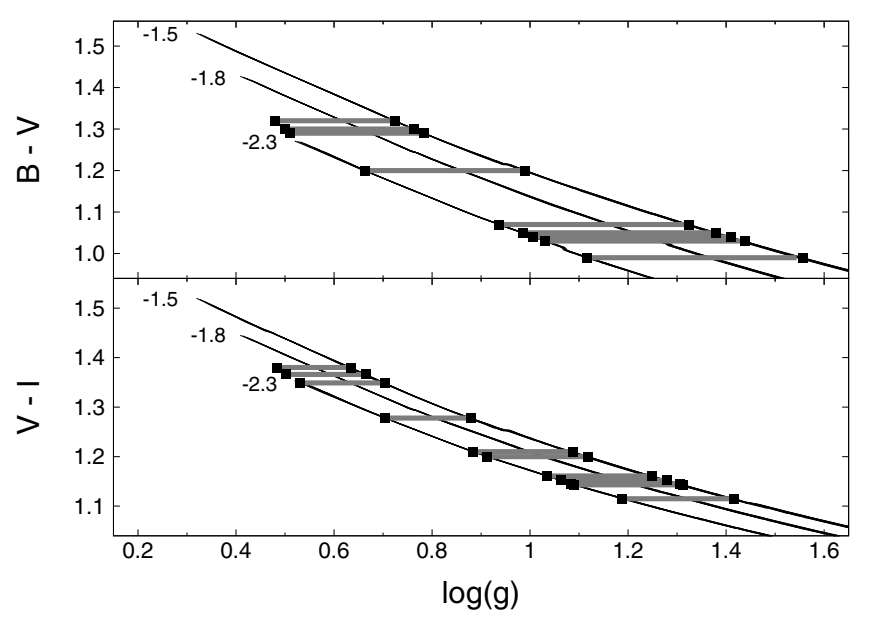

Fig. 9. Estimation of the gravity of the M53 giants from colors by using the evolution models of Pietrinferni et al. (2004). Overall iron abundances are shown to the left of the isochrones spreading from 10 to $13.5 \mathrm{Gyr}$ (note that the age dependence is highly degenerate). Horizontal bars denote the positions of the giants as listed in Table A.1.

solar-type heavy element distribution), we can estimate $[\mathrm{Fe} / \mathrm{H}]$ by using $B V I$ magnitudes, assuming that we can give a reasonable estimate of $\log g$. For Cepheids and RR Lyrae stars this estimate is very accurate, since the period is in tight correlation with the gravity. For giants we can obtain estimates on the possible ranges of the gravity by employing evolutionary models. We took the isochrones from the evolutionary models of Pietrinferni et al. (2004) with metallicities bracketing the estimated metallicity of -2.0 dex. Then, we checked the position of the selected giants (see Appendix A) with available three-color photometry on the $\log g \rightarrow(B-V),(V-I)$ diagrams. We then took the corresponding $\log g$ ranges and computed individual metallicities from the stellar atmosphere models of Castelli (1999).

The relation between $\log g$ and the color indices at various metallicity values and cluster ages is shown in Fig. 9. It is very fortunate that there is basically no age-dependence in this diagram, so one can read off the corresponding $\log g$ ranges affected only by the adopted metallicity values. It turns out that in the color index $-\log g$ space of the giants studied, there is a very low dependence of the derived metallicity on the assumed values of $\log g$. (We note, however, that for $\log g$ values exceeding the high-metallicity bounds by $\sim 0.3-0.5$ dex we could see a significant increase of up to $0.5 \mathrm{dex}$ in $[\mathrm{Fe} / \mathrm{H}]$.) With the $\log g$ ranges confined in this way, we computed more accurate individual photometric abundances by matching both color indices simultaneously. The derived values, together with other important data on the stars used, are given in Table A.1.

For comparison with the values obtained on RRab stars by using the empirical formula of JK96, we plotted the metallicities for the two groups of stars in Fig. 10. Error bars of the individual RGB stars denote standard deviations, obtained by adding uncorrelated Gaussian random noise with $\sigma=0.02$ mag to their $B-V$ and $V-I$ color indices (the very small formal errors for the RRab stars are not shown). In spite of the large scatter, it is clear that the RGB stars have significantly lower overall metallicities. Indeed, the average metallicities with their formal $1 \sigma$ errors are $-2.12 \pm 0.05$ and $-1.58 \pm 0.03$, i.e., the difference is well above the $3 \sigma$ level. We note that our average photometric metallicity shows close agreement with the ones obtained by spectroscopic measurements, so the suspected systematic difference between the HB and RGB populations seems to be confirmed. However, 


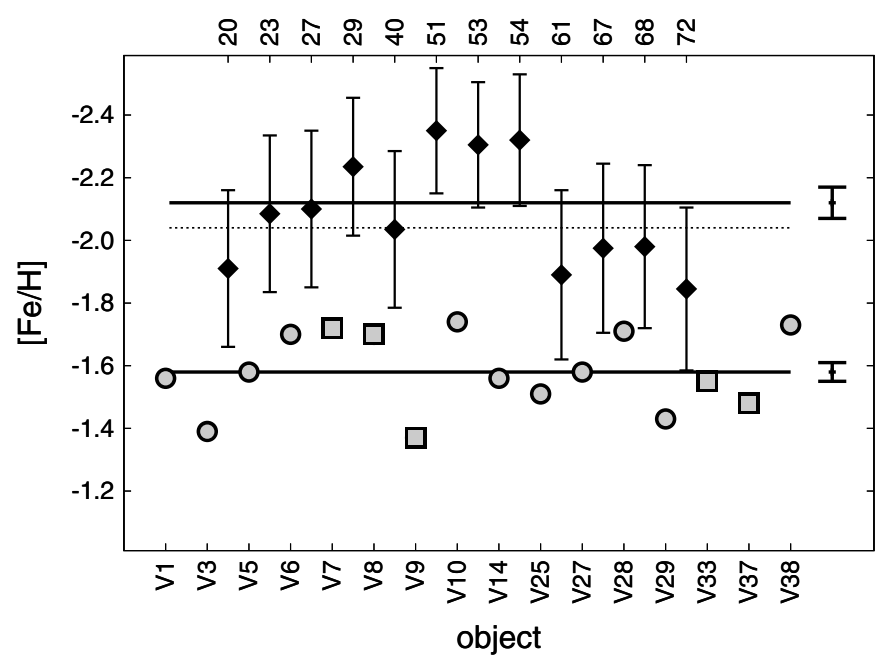

Fig. 10. Photometrically derived overall iron abundances of 12 RGB stars of M53 (black diamonds) versus iron abundances of $16 \mathrm{RRab}$ stars computed from the $V$ light curves by the JK96 formula (gray circles [data from this paper] and squares [data from Kopacki 2000]). Horizontal lines show the corresponding averages (for comparison, we also show the value given by Zinn \& West 1984 with a dashed line). Formal $1 \sigma$ errors are given for the means. For star IDs we refer to Tables 1 and A.1.

we do not know at this point what the source of this difference is. Although we cannot exclude that there are substantial chemical inhomogeneities in some clusters (e.g., NGC 1851, see Lee et al. 2009), we think that it is more probable that the JK96 formula is biased upward at lower metallicities due to the low number of low-metallicity stars in the calibration sample (see also Schwarzenberg-Czerny \& Kaluzny 1998; Kovács 2002; Nemec 2004). To check if this is indeed the case, we would need either precise $B V I$ photometry, or, even better, high-dispersion spectroscopy for the RR Lyrae stars. Unfortunately, there are no such data available. We made an attempt to utilize some of the sporadic multicolor data for, e.g., M15, M68, and M92 (Corwin et al. 2008; Walker 1994; Kovács \& Jurcsik 1997 and references therein) but either the number of objects was low or the average magnitudes were too poor to derive consistent metallicities.

Since there are ample amounts of RRc stars in M53, we decided to employ the formula calibrated by Morgan et al. (2007) for an independent estimation of the metallicity of the RR Lyrae population. By using their Eq. (3) and transforming the values to the scale of Jurcsik (1995), we find an average $[\mathrm{Fe} / \mathrm{H}]$ of $-1.68 \pm 0.08$ (error of the mean) for the sample of 15 stars (for two stars, data were taken from Kopacki 2000). Although this value confirms the one derived from the RRab population, we note the following: (i) the structure of the RRc light curves contain fewer distinct features than those of the RRab stars and even these are often washed out by noise; (ii) the calibration for the RRc stars is based on cluster variables that, because of the absence of individual metallicity estimates, are assumed to have the same metallicity as that of the host cluster (i.e., there are 12 independent metallicity values used in the regression for the 106 variables in the calibrating sample); because of the use of cluster variables, the full span of the metallicity range is only $[-1.0,-2.2]$, that is nearly a factor of two smaller than the one used by JK96 for the Galactic field RRab stars. We presume that the good overall agreement between the metallicities is primarily due to the strong dependence of $[\mathrm{Fe} / \mathrm{H}]$ on the period for both types of stars.
We made yet another test of the possible dependence of the derived metallicity on the chemical composition (namely on $\alpha$ element enhancement, since the cluster is of low metallicity). It turned out that the $\alpha$-enhanced models shift the lower boundary of the $\log g$ range downward by some $\sim 0.2$ dex and resulted in a small (i.e., $<0.1 \mathrm{dex}$ ) and apparently non-systematic change in the metallicity $[\mathrm{M} / \mathrm{H}]$. We recall that if the solution of the discrepancy between the JK96 and RGB metallicities lies in the non-solar chemical composition, then we would expect a dramatic increase in the overall RGB metallicity $[\mathrm{M} / \mathrm{H}]$ estimated by the above method from the $\alpha$-enhanced models, since then the relative abundance of iron (to which the JK96 formula is calibrated) would be lower in these models.

Based on the above results we can check how the cluster's distance, as derived from the PLC relation, relates to the one estimated from the more traditional (but less accurate, due to evolutionary effects, see, e.g., Cassisi et al. 2004) $M_{V}-[\mathrm{Fe} / \mathrm{H}]$ relation calibrated by the same BW distance scale. Relying on the same BW sample as in Sect. 3.2, and using the same spectroscopic metallicities as in Dékány et al. (2008) we obtain the following linear calibration between the absolute $V$ magnitude and the iron abundance:

$M_{V}=(0.24 \pm 0.06)[\mathrm{Fe} / \mathrm{H}]_{\mathrm{JK} 96}+(0.88 \pm 0.06)$.

As expected, the standard deviation of the above fit is very large (0.17 mag compared to $0.063 \mathrm{mag}$ of the PLC relation - see Sect. 3.2). Although many variants of $M_{V}-[\mathrm{Fe} / \mathrm{H}]$ calibrations can be found in the literature, the slope of the above formula turns out to be very close to the value of $0.23 \pm 0.04$, favored by recent reviews on this subject (see, e.g., Catelan 2009, and references therein). The mean $V$ magnitude of M53 RR Lyrae stars is equal to $16.85 \pm 0.01 \mathrm{mag}$, based on the magnitude-transformed light curves of 24 stars (see Table 1). Using the two different metallicity values derived for the RR Lyrae and red giant stars, from Eq. (3) we get $16.35 \pm 0.11$ and $16.48 \pm 0.14$, respectively, for the distance modulus of the cluster. Both are in agreement with the PLC result in Sect. 3.2 within the errors, but with an apparent preference for higher metallicity.

\section{Short-period variables}

We Fourier-analyzed the photometric time-series of all identified objects in the $[0,50] \mathrm{d}^{-1}$ frequency range to search for short periodic variables, in particular for SX Phoenicis and high-amplitude Delta Scuti stars. With the aid of TFA, we considerably increased the reliability of detection of short-period, low-amplitude signals by filtering out low-frequency trends from the data whose amplitudes would have overwhelmed the intrinsic light variations of these objects.

All of the short-period stars previously known in M53 were discovered by Jeon et al. (2003) who used a 1.8-m telescope and PSF photometry. All of these objects are of SX Phe-type located in the blue straggler region of the color-magnitude diagram (CMD). We successfully identified SXP1, SXP2, SXP6, and SXP7 among these eight objects in our data (they are identical to our V73, V74, V75, and V76, respectively). From the data published by Jeon et al. (2003), SXP4, SXP5, and SXP8 have too low amplitudes to be identified in our data. The situation is different for SXP3 which has a reasonably large amplitude of $0.1 \mathrm{mag}$ according to Jeon et al. (2003) but it is rather close to V66 and V67 and the limited resolution of our CCD chip did not enable us to separate their light variations.

In addition to the previously known SX Phe stars, we discovered seven new objects (denoted V77 through V83) that have 
Table 3. Properties of the short period stars.

\begin{tabular}{lccrrr}
\hline \hline ID & $\alpha[\mathrm{hms}]$ & $\delta[\mathrm{dms}]$ & $d\left[^{\prime \prime}\right]$ & $f\left[\mathrm{~d}^{-} 1\right]$ & $S N R$ \\
\hline V73 & $13: 13: 03.1$ & $18: 09: 26.1$ & 119.9 & 14.26581 & 10.2 \\
V74 & $13: 12: 49.7$ & $18: 07: 26.3$ & 181.9 & 22.03949 & 13.7 \\
V75 & $13: 13: 09.4$ & $18: 09: 39.5$ & 203.9 & 22.60022 & 11.8 \\
V76 & $13: 13: 05.0$ & $18: 08: 35.3$ & 168.4 & 24.10224 & 10.4 \\
V77* & $13: 13: 20.8$ & $18: 15: 34.9$ & 488.3 & 13.00995 & 23.0 \\
V78* & $13: 12: 49.8$ & $18: 08: 56.0$ & 106.9 & 22.25488 & 12.7 \\
V79* & $13: 12: 46.6$ & $18: 11: 36.9$ & 150.6 & 21.59184 & 9.7 \\
\hline V80* & $13: 12: 57.4$ & $18: 10: 13.8$ & 31.3 & 14.83029 & 14.2 \\
V81* & $13: 13: 02.7$ & $18: 06: 29.4$ & 244.5 & 14.01180 & 9.5 \\
V82* & $13: 12: 56.5$ & $18: 13: 10.0$ & 181.1 & 45.33103 & 8.4 \\
V83* & $13: 12: 50.1$ & $18: 07: 42.9$ & 164.5 & 8.01907 & 8.2 \\
\hline
\end{tabular}

Notes: new discoveries are marked with asterisks. The signal-to-noise ratio $(\mathrm{SNR})$ was calculated in the $[0,50] \mathrm{d}^{-1}$ frequency range. See Table 1 for other notations.

significant high-frequency signals. Except for two of them, these faint objects were heavily crowded or merged with brighter stars, therefore, we were not able to obtain accurate $R F$ magnitudes for them to perform the flux-to-magnitude conversion. To investigate if these objects were located in the blue straggler star (BSS) region, i.e., they could be securely classified as SX Phe stars, we sought for supplementary information in the literature. We cross-correlated the positions of the 7 new objects with the single-epoch data-base of M53 BSSs by Beccari et al. (2008a, via private comm.) and found a match with sub-pixel accuracy in three cases, namely $\mathrm{V} 77, \mathrm{~V} 78$, and V79, which suggests that these variables are most probably of SX Phe type. For V77 $(V=18.478, V-I=0.433)$ and V81 $(V=17.454$, $V-I=0.815$ ), we were able to perform the conversion of fluxes into magnitudes. The average magnitudes of V77 verifies its location in the BSS region, while V81 is located exactly on the lower red giant branch (see the CMD of Beccari et al. 2008a). We are not sure whether there is a blending effect of two stars in this case or V81 (which we identified as USNO-B1.0 10810245849 ) is indeed a peculiar variable. We note however that there is a suspected BSS (USNO-B1.0 1081-0245846) near V81 which might account for this variability, although we did not detect the corresponding signal in its time-series.

The type of the remaining three new short-period variables (V80, V82, and V83) remains unknown, but their blue straggler nature cannot be ruled out (for example, we note that the previously known variable V73 was not identified as a BSS by Beccari et al. 2008a). The properties of all 11 short-period variables detected in this study are given in Table 3. The light curves and amplitude spectra of the objects are shown in Figs. 11-12.

\section{Long-period variables}

Most of the stars on the upper part of the giant branch of M53 show some kind of low-amplitude light variations on time-scales of greater than $10 \mathrm{~d}$. We identified V65, V66, V67, V69, and V70 (see the CVSGC for their positions) among the previously known variable red giants, all of them showing a large power excess at very low frequencies $\left(\sim 0.01 \mathrm{~d}^{-1}\right)$, implying long-term irregular variations.

At the same time, we found three new long-period variables (LPVs) among the red giant stars (signed as V84, V85, and V86). The properties of these new objects are summarized in Table 4 and their light curves are shown in Fig. 13. Variable V86 was saturated in the $I$ band, therefore we give only an approximate $V$ magnitude in Table 4 as a reference. V84 and V86 have very

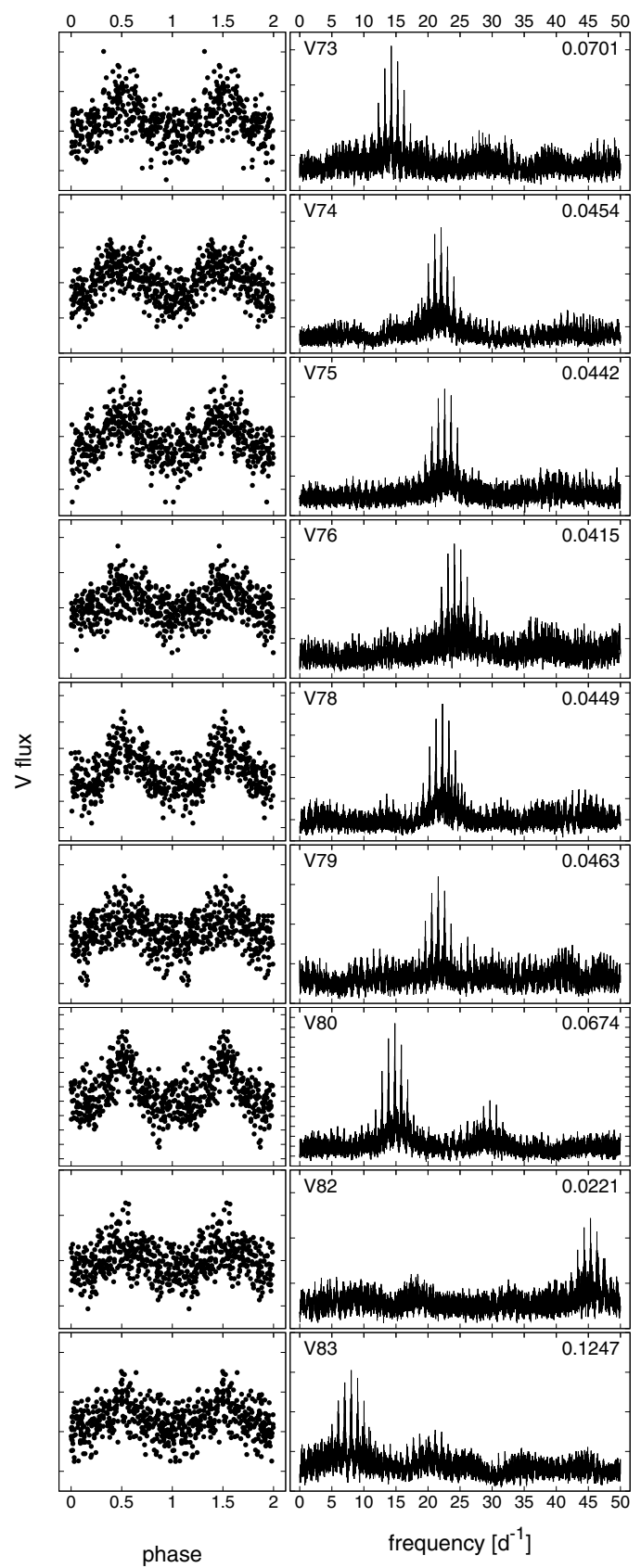

Fig. 11. Left panels: TFA-reconstructed and folded light curves of the short period stars. Right panels: DFT amplitude spectra of the corresponding time-series. The separation between ordinate ticks denotes the same flux difference in each plot. IDs and periods are given in each case. See Table 3 for additional properties of these variables.

similar periods. We excluded the possibility of detecting the same systematics in these objects by using a large variety of template sets (see Sect. 2.2) and also by verifying that the two light variations are not in the same phase.

Recent investigations based mainly on OGLE-II data have showed that most of the pulsating red giants below the tip of the red giant branch (TRGB) are among the first ascent RGB stars (see e.g., Kiss \& Bedding 2003). The majority of these stars lie on parallel ridges in the period-luminosity (PL) plane. We inspected whether the above two LPVs with $V-I$ color indices match a PL ridge on the $\log P$-Wesenheit index plane of LMC giants by Soszyński et al. (2005), assuming an LMC distance modulus of 18.5. We found that V84 is below the faint limit of 


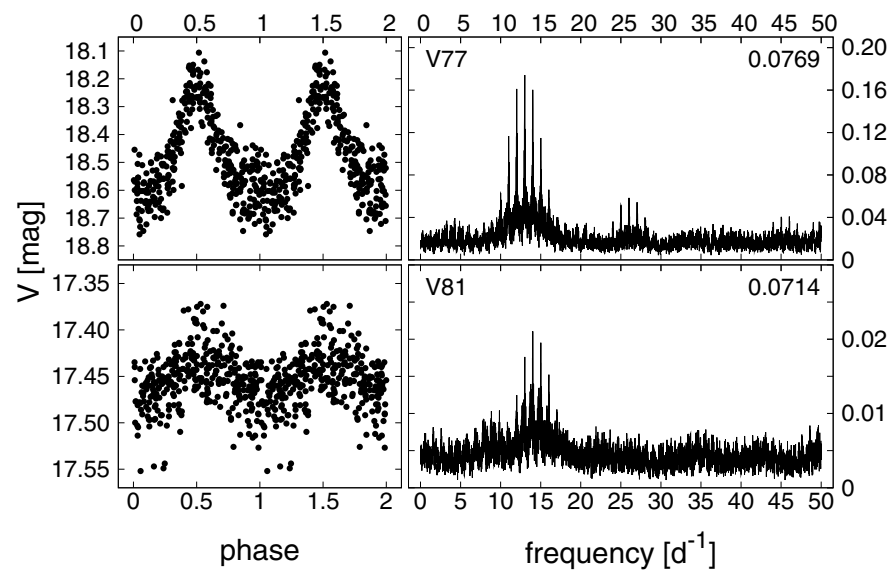

Fig. 12. TFA-reconstructed light curves and DFT amplitude spectra of V77 and V81. Labeling is the same as in Fig. 11.

Table 4. Properties of the long period variables.

\begin{tabular}{llcccccc}
\hline \hline ID & $\alpha[\mathrm{hms}]$ & $\delta[\mathrm{dms}]$ & $d\left[{ }^{\prime \prime}\right]$ & $P[\mathrm{~d}]$ & $S N R$ & $V$ & $V-I$ \\
\hline V84 & $13: 12: 36.2$ & $18: 07: 32.3$ & 306.6 & 22.4 & 15.5 & 14.766 & 1.175 \\
V85 & $13: 12: 50.7$ & $18: 10: 39.7$ & 69.7 & 19.8 & 19.2 & 13.962 & 1.357 \\
V86 & $13: 12: 52.7$ & $18: 10: 28.0$ & 39.4 & 22.2 & 12.9 & $\sim 14.2$ & $\ldots$ \\
\hline
\end{tabular}

Notes: for variable V86, only instrumental $V$ magnitude average is shown because the star was saturated in the $I$ band. The signal-to-noise ratio (SNR) was calculated in the $[0,20] \mathrm{d}^{-1}$ frequency range.

the LMC ridges, while V85 shows a close match to ridge $A / R_{3}$ which was associated with third-overtone pulsators by Wood (2000).

\section{Conclusions}

The globular cluster M53 was observed with the prime goal of extending the available data-base of variable stars. This is the first time-series photometric study covering the whole cluster up to $\sim 14^{\prime}$ (for a comparison, the tidal radius of the cluster is $\sim 22^{\prime}$, according to Harris 1996). We analyzed the whole set of $V, I$ photometric time-series data acquired on 3048 objects in two seasons in 2007-2008. Our goal was to establish a reasonably deep variable star inventory by considering all possible variable types that may occur throughout the Hertzsprung-Russell diagram. Because of the limited time span of the observations, the search was limited to periods $\lesssim 100$ days. Similarly, the lower bound of the brightness level was limited by the telescope aperture and overall sky conditions to $V \lesssim 21 \mathrm{mag}$, including the blue straggler region and ending $\sim 1$ mag below the main sequence turn-off point (e.g., Rey et al. 1998). In analyzing the data we used the OIS method for image processing and TFA for time-series post-processing.

Altogether 12 new variables were identified, most of them are of short period (three are of SX Phe-type). Interestingly, and somewhat surprisingly, except for the three Blazhko variables, we did not find any multiperiodic variables, although they could be quite abundant among RR Lyrae and SX Phe stars (Kovács 2001; Gilliland et al. 1998). We were also unsuccessful in finding eclipsing binaries, although some $10 \%$ of the blue stragglers are thought to form binary systems (Beccari et al. 2008b). We note that in NGC 5466, a similarly low metallicity cluster, Arellano Ferro et al. (2008) identified three eclipsing variables discovered by Mateo et al. (1990). They are all in the BSS region. It may well be that the relatively high scatter of our data at their low brightness level prevented us from detecting these types of binaries. It is interesting to note that we did not find variables slightly more luminous than the horizontal branch near the red giants. In a similar analysis of a sample of the Large Magellanic Cloud field stars, Szulágyi et al. (2009) found several such stars (see also Arellano Ferro et al. 2008 for two such stars, one is right on the red giant branch of NGC 5466). We note, however, that we have four short period stars with periods reminiscent of the SX Phe stars. Although, except for one object, we do not have color information on these, based on their very short periods of less than 0.13 days, we suspect that all of them are actually SX Phe variables.

In analyzing the variables identified in the present data, we focused on the RR Lyrae stars. Two important parameters (average distance and metallicity) that are relevant for the physical properties of the cluster were derived from the light curves and average colors. We have shown that the period-luminosity-color (PLC) relation spanned by the RRab stars as observed in other globular clusters, is also very clear in M53. Furthermore, it was also demonstrated for the first time on an empirical basis that RRc (first overtone RR Lyrae) stars, after a proper period shift, follow the same linear $\log P_{0}-W(V-I)$ relation as those of the RRab (fundamental mode) stars. By using the Baade-Wesselink calibration of this relation (Kovács 2003), we derived the distance modulus of $16.31 \pm 0.04$ mag for the cluster.

By using the iron abundance-Fourier parameter relation of Jurcsik \& Kovács (1996), we derived an overall iron abundance of $-1.58 \pm 0.03$ dex for the RRab stars. This is significantly higher than the value of -2.1 computed from lowdispersion spectra for the red giant stars. The lower metallicity of the giants has been confirmed by our estimate based on $B V I$ photometry, stellar atmosphere and evolution models. Such a discrepancy of $0.3-0.5$ dex seems to be present also in other lowmetallicity clusters. For example, for the RRab stars of M68 we find $[\mathrm{Fe} / \mathrm{H}]=-1.8$ (Jurcsik \& Kovács 1996), whereas the highdispersion spectroscopic data on giants by Lee et al. (2005) suggest a value of -2.2 dex (both values are on the scale of Jurcsik 1995). Similarly, for the RRab stars of NGC 5053, Nemec (2004) finds $[\mathrm{Fe} / \mathrm{H}]=-1.7$, whereas the low-dispersion spectral indices of Suntzeff et al. (1988) yield -2.3 dex for the giants.

Our current understanding of this discrepancy lies in the lack of sufficient low-metallicity RRab stars in the original calibration set of Jurcsik \& Kovács (1996). Unfortunately, from the works of Suntzeff et al. (1994) and that of Layden (1994) there were no new efforts to obtain metal abundances for a large number of field stars. A reliable re-calibration of the formula cannot be made without a new and homogeneous sample. As an example of the need for a new spectroscopic survey, we mention the case of UU Boo (Jurcsik et al. 2008), for which the current Fourier formula yields $[\mathrm{Fe} / \mathrm{H}]=-1.17$, whereas the spectroscopic observations of Layden (1994) and Kinman \& Carretta (1992) resulted in values of -1.64 and -1.00 , respectively. Although other tests based on new and accurate measurements of non-Blazhko stars show remarkable agreement with earlier spectroscopic values (e.g., Kun et al. 2008), the above example strongly supports the need to revisit Galactic field RR Lyrae stars by high-dispersion spectroscopic surveys. Within the same framework, a more thorough discussion of the RRab giant metallicity discrepancy would require a systematic highdispersion spectroscopic study of the RR Lyrae and other nonvariable populations of globular clusters. Using the same type of measurements of all cluster members selected and analyzing the 

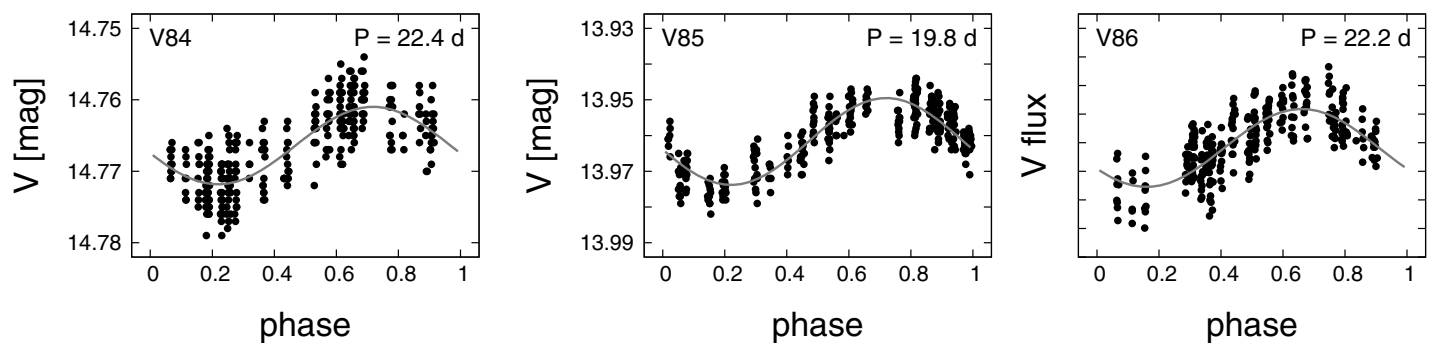

Fig. 13. $V$ light curves of the 3 new long-period red giants folded with their periods. Star IDs, periods, and sine curves fitted to the data are also shown.

Table A.1. Properties of the RGB stars.

\begin{tabular}{lccrcccc}
\hline \hline ID & $\alpha[\mathrm{hms}]$ & $\delta[\mathrm{dms}]$ & \multicolumn{1}{c}{$d\left[^{\prime \prime}\right]$} & $V$ & $B-V$ & $V-I$ & {$[\mathrm{Fe} / \mathrm{H}]_{B V I}$} \\
\hline 20 & $13: 12: 56.9$ & $18: 08: 41.9$ & 91.2 & 14.04 & 1.30 & 1.35 & $-1.91 \pm 0.25$ \\
23 & $13: 13: 03.7$ & $18: 09: 38.7$ & 123.9 & 14.07 & 1.32 & 1.38 & $-2.09 \pm 0.25$ \\
27 & $13: 12: 37.4$ & $18: 08: 23.4$ & 276.5 & 14.08 & 1.32 & 1.38 & $-2.10 \pm 0.25$ \\
29 & $13: 12: 50.4$ & $18: 08: 53.7$ & 102.6 & 14.11 & 1.29 & 1.37 & $-2.24 \pm 0.22$ \\
40 & $13: 12: 48.6$ & $18: 14: 15.7$ & 263.5 & 14.34 & 1.20 & 1.28 & $-2.04 \pm 0.25$ \\
51 & $13: 13: 17.4$ & $18: 14: 46.0$ & 419.4 & 14.56 & 1.08 & 1.21 & $-2.35 \pm 0.20$ \\
53 & $13: 12: 48.2$ & $18: 12: 46.6$ & 186.3 & 14.59 & 1.08 & 1.20 & $-2.31 \pm 0.20$ \\
54 & $13: 13: 05.3$ & $18: 14: 50.8$ & 315.7 & 14.62 & 1.07 & 1.20 & $-2.32 \pm 0.21$ \\
61 & $13: 12: 55.6$ & $18: 13: 13.8$ & 184.2 & 14.74 & 1.05 & 1.15 & $-1.89 \pm 0.27$ \\
67 & $13: 12: 38.3$ & $18: 11: 04.7$ & 248.0 & 14.80 & 0.99 & 1.12 & $-1.98 \pm 0.27$ \\
68 & $13: 13: 02.5$ & $18: 13: 23.2$ & 219.2 & 14.83 & 1.03 & 1.15 & $-1.98 \pm 0.26$ \\
72 & $13: 12: 47.9$ & $18: 06: 32.2$ & 241.5 & 14.88 & 1.04 & 1.14 & $-1.85 \pm 0.26$ \\
\hline
\end{tabular}

Note: ID numbers and $B-V$ values were taken from the catalog of Rey et al. (1998). Photometric metallicities $[\mathrm{Fe} / \mathrm{H}]_{B V I}$ were derived as described in Sect. 3.3.

chemical composition in the same way would lead to a uniform treatment of their composition and, in particular, to the avoidance of the ongoing confusion about the different zero points and scales (i.e., Zinn \& West 1984 vs. high-dispersion values). The importance of a more sophisticated treatment of the metallicity issue is underlined by current works on detailed abundance analyses of globular cluster stars (e.g., Lee et al. 2009) and on individual field stars (e.g., Preston et al. 2006; Wallerstein et al. 2009).

\section{Appendix A: Red giant stars}

For the estimation of $[\mathrm{Fe} / \mathrm{H}]$ using the photometric method described in Sect. 3.3 we needed accurate $B-V$ and $V-I$ color indices for a number of red giant stars in M53. Since our observations covered only the $V$ and $I$ bands, we took the $B-V$ values from the catalog of Rey et al. (1998). We selected a large number of stars from this catalog with $V$ and $B-V$ values consistent with an RGB evolutionary state according to the color-magnitude diagram published by Rey et al. (1998). We mapped the instrumental pixel positions of the catalog onto our reference system by using the positions of RR Lyrae stars common in Rey's catalog and our data-base. This transformation yielded positions with an accuracy of a few arcseconds which was sufficient to unambiguously identify these bright stars in our frames by eye. As a further selection procedure we rejected the saturated stars and kept only those that were well outside the core and therefore had reasonably low photometric errors on the RFs. After the flux-to-magnitude transformation, we computed the simple $V-I$ magnitude averages for these objects. We note that almost all RGB objects in our sample showed some kind of low amplitude irregular light variations on long timescales. Therefore we rejected those objects for which the difference was too high between our $V$ magnitude and that of Rey et al. (1998). In our final sample of 12 RGB stars the standard deviation in the differences of the two $V$ magnitudes is $0.02 \mathrm{mag}$. Table A.1 lists catalog IDs, coordinates, distances from the cluster center, magnitudes, and estimated $[\mathrm{Fe} / \mathrm{H}]$ values for these 12 red giants.

Acknowledgements. We are very grateful to Dr. Giacomo Beccari for providing us with his data-base of M53 blue straggler stars. We are indebted to the editor Dr. Thierry Forveille for the patient mediation between the referees and the authors. We thank the anonymous second referee for the quick and constructive comments. We acknowledge the support of the Hungarian Scientific Research Fund (OTKA, grant No. K-60750). This research has made use of the VizieR catalog access tool, CDS, Strasbourg, France.

\section{References}

Alard, C. 2000, A\&AS, 144, 363

Alard, C., \& Lupton, R. H. 1998, ApJ, 503, 325

Arellano Ferro, A., Rojas López, V., Giridhar, S., et al. 2008, MNRAS, 384, 1444

Bakos, G. Á., Torres, G., Pál, A., et al. 2009, ApJ, submitted [arXiv: 0901.0282]

Beccari, G., Lanzoni, B., Ferraro, F. R., et al. 2008a, ApJ, 679, 712

Beccari, G., Pulone, L., Ferraro, F. R., et al. 2008b, Mem. Soc. Astron. Ital., 79, 360

Bono, G., Caputo, F., Castellani, V., Marconi, M., \& Storm, J. 2001, MNRAS, 326,1183

Cáceres, C., \& Catelan, M. 2008, ApJS, 179, 242

Cardelli, J. A., Clayton, G. C., \& Mathis, J. S. 1989, ApJ, 345, 245

Cassisi, S., Castellani, M., Caputo, F., et al. 2004, A\&A, 426, 641

Castelli, F. 1999, A\&A, 346, 564

Catelan, M. 2009, Ap\&SS, (tmp) 18

Clement, C. M., Muzzin, A., Dufton, Q., et al. 2001, AJ, 122, 2587

Cortés, C., \& Catelan, M. 2008, ApJS, 177, 362

Corwin, T. M., Borissova, J., Stetson, P. B., et al. 2008, AJ, 135, 1459

Cox, A. N., Hodson, S. W., \& Clancy, S. P. 1983, ApJ, 266, 94

Dékány, I., Kovács, G., Jurcsik, J., et al. 2008, MNRAS, 386, 521

di Benedetto, G. P. 2008, MNRAS, 390, 1762

Di Criscienzo, M., Marconi, M., \& Caputo, F. 2004, ApJ, 612, 1092

Garcia Lugo, G., Arellano Ferro, A., \& Rosenzweig, P. 2007, in IAU Symp. 240, ed. W. I. Hartkopf, E. F. Guinan, \& P. Harmanec, 214

Gilliland, R. L., Bono, G., Edmonds, P. D., et al. 1998, ApJ, 507, 818

Harris, W. E. 1996, AJ, 112, 1487

Jeon, Y.-B., Lee, M. G., Kim, S.-L., et al. 2003, AJ, 125, 3165

Jurcsik, J. 1995, Acta Astron., 45, 653

Jurcsik, J., \& Kovács, G. 1996, A\&A, 312, 111

Jurcsik, J., Sódor, A., Hurta, Z., et al. 2008, IBVS, 5844

Kinemuchi, K., Harris, H. C., Smith, H. A., et al. 2008, AJ, 136, 1921

Kinman, T. D., \& Carretta, E. 1992, PASP, 104, 111

Kiss, L. L., \& Bedding, T. R. 2003, MNRAS, 343, L79

Kopacki, G. 2000, A\&A, 358, 547

Kovács, G. 2001, The multiperiodic behavior of RR Lyrae stars, ed. M. Takeuti, \& D. D. Sasselov, 61

Kovács, G. 2002, in Omega Centauri, A Unique Window into Astrophysics, ed. F. van Leeuwen, J. D. Hughes, \& G. Piotto, ASPCS, 265, 163

Kovács, G. 2003, MNRAS, 342, L58

Kovács, G. 2008, to appear in the proceedings of the "Nonlinear stellar hydrodynamics and the pulsations of Cepheids" workshop, ed. M.-J. Goupil, Z. Kolláth, P. Kervella, N. Nardetto, EAS Publ. Ser., 38, 2009, 91 
Kovács, G., \& Bakos, G. Á. 2007, in Transiting Extrapolar Planets Workshop, ed. C. Afonso, D. Weldrake, \& T. Henning, ASPCS, 366, 133

Kovács, G., \& Bakos, G. A. 2008, CoAst, 157, 82

Kovács, G., \& Jurcsik, J. 1997, A\&A, 322, 218

Kovács, G., \& Walker, A. R. 2001, A\&A, 374, 264

Kovács, G., Zucker, S., \& Mazeh, T. 2002, A\&A, 391, 369

Kovács, G., Bakos, G., \& Noyes, R. W. 2005, MNRAS, 356, 557

Kun, E., Sódor, A., Jurcsik, J., et al. 2008, IBVS, 5859

Lasker, B. M., Lattanzi, M. G., McLean, B. J., et al. 2008, AJ, 136, 735

Layden, A. C. 1994, AJ, 108, 1016

Lee, J.-W., Carney, B. W., \& Habgood, M. J. 2005, AJ, 129, 251

Lee, J.-W., Lee, J., Kang, Y.-W., et al. 2009, ApJ, 695, L78

Longmore, A. J., Dixon, R., Skillen, I., Jameson, R. F., \& Fernley, J. A. 1990, MNRAS, 247, 684

Marín-Franch, A., Aparicio, A., Piotto, G., et al. 2009, ApJ, 694, 1498

Mateo, M., Harris, H. C., Nemec, J., et al. 1990, AJ, 100, 469

Morgan, S. M., Wahl, J. N., \& Wieckhorst, R. M. 2007, MNRAS, 374, 1421

Nagy, A., \& Kovács, G. 2006, A\&A, 454, 257

Nemec, J. M. 2004, AJ, 127, 2185

Pietrinferni, A., Cassisi, S., Salaris, M., et al. 2004, ApJ, 612, 168

Pilachowski, C. A., Bothun, G. D., Olszewski, E. W., et al. 1983, ApJ, 273, 187
Preston, G. W., Thompson, I. B., Sneden, C., Stachowski, G., \& Shectman, S. A. 2006, AJ, 132, 1714

Rey, S.-C., Lee, Y.-W., Byun, Y.-I., et al. 1998, AJ, 116, 1775

Schlegel, D. J., Finkbeiner, D. P., \& Davis, M. 1998, ApJ, 500, 525

Schwarzenberg-Czerny, A., \& Kaluzny, J. 1998, MNRAS, 300, 251

Shapley, H. 1920, ApJ, 52, 73

Soszyński, I., Udalski, A., Szymanski, M., et al. 2003, Acta Astron., 53, 93

Soszyński, I., Udalski, A., Kubiak, M., et al. 2005, Acta Astron., 55, 331

Stetson, P. B. 1987, PASP, 99, 191

Stetson, P. B. 2000, PASP, 112, 925

Suntzeff, N. B., Kraft, R. P., \& Kinman, T. D. 1988, AJ, 95, 91

Suntzeff, N. B., Kraft, R. P., \& Kinman, T. D. 1994, ApJS, 93, 271

Székely, P., Kiss, L. L., Jackson, R., et al. 2007, A\&A, 463, 589

Szulágyi, J., Kovács, G., \& Welch, D. L. 2009, A\&A, 500, 917

Udalski, A., Szymanski, M., Kubiak, M., et al. 1999, Acta Astron., 49, 201

van den Hoven van Genderen, E. 1947, Bull. Astron. Inst. Netherlands, 10, 241

Walker, A. R. 1994, AJ, 108, 555

Wallerstein, G., Kovtyukh, V. V., \& Andrievsky, S. M. 2009, ApJ, 692, L127

Wood, P. R. 2000, PASA, 17, 18

Wozniak, P. R. 2000, Acta Astron., 50, 421

Zinn, R. 1985, ApJ, 293, 424

Zinn, R., \& West, M. J. 1984, ApJS, 55, 45 
I. Dékány and G. Kovács: Study of M53, Online Material $p 1$

Table 1. Properties of the RR Lyrae stars.

\begin{tabular}{|c|c|c|c|c|c|c|c|c|}
\hline$\overline{\text { ID }}$ & Type & $\overline{\alpha \alpha[\mathrm{hms}]}$ & $\delta$ [dms] & $d\left[^{\prime \prime \prime}\right]$ & $\overline{P[\mathrm{~d}]}$ & $\overline{S S N R}$ & $\bar{V}$ & $\overline{V-I}$ \\
\hline V1 & RRab & $13: 12: 56.3$ & $18: 07: 13.8$ & 176.8 & $0.6098298(9)$ & 14.3 & 16.970 & 0.579 \\
\hline V2 & $\mathrm{RRc}$ & $13: 12: 50.3$ & 18:07:00.8 & 202.0 & $0.386122(1)$ & 22.2 & 16.879 & 0.402 \\
\hline V3 & RRab & $13: 12: 51.4$ & $18: 07: 45.5$ & 154.6 & $0.630605(1)$ & 17.8 & 16.848 & 0.447 \\
\hline V4 & $\mathrm{RRc}$ & $13: 12: 43.9$ & $18: 07: 26.4$ & 230.3 & $0.385545(1)$ & 26.0 & 16.800 & 0.383 \\
\hline V5 & RRab & $13: 12: 39.1$ & $18: 05: 42.6$ & 353.3 & $0.639426(1)$ & 23.2 & 16.888 & 0.526 \\
\hline V6 & RRab & $13: 13: 03.9$ & 18:10:19.8 & 123.7 & $0.664020(1)$ & 14.4 & 16.819 & 0.522 \\
\hline V7 & RRab & $13: 13: 00.9$ & $18: 11: 29.7$ & 113.3 & $0.5448584(6)$ & 13.5 & $\ldots$ & $\ldots$ \\
\hline V8 & RRab & $13: 13: 00.4$ & 18:11:05.1 & 92.3 & $0.615528(1)$ & 16.1 & $\ldots$ & $\ldots$ \\
\hline V9 & RRab & 13:13:00.1 & $18: 09: 25.0$ & 81.9 & $0.6003690(7)$ & 15.2 & & \\
\hline V10 & RRab & $13: 12: 45.7$ & $18: 10: 55.6$ & 143.3 & $0.6082612(7)$ & 18.8 & 16.837 & 0.463 \\
\hline V11 & RRabB & $13: 12: 45.4$ & 18:09:01.9 & 156.3 & $0.629940(5)$ & 12.2 & . & \\
\hline V14 & RRab & $13: 13: 20.5$ & $18: 06: 42.8$ & 415.6 & $0.5454625(7)$ & 14.0 & 16.880 & 0.452 \\
\hline V15 & $\mathrm{RRc}$ & $13: 13: 12.4$ & $18: 13: 55.0$ & 332.5 & $0.3086646(9)$ & 21.6 & 16.894 & 0.342 \\
\hline V16 & RRcB & $13: 12: 46.2$ & $18: 06: 39.2$ & 247.3 & $0.3031686(7)$ & 18.7 & $\cdots$ & \\
\hline V17 & $\mathrm{RRc}$ & $13: 12: 40.4$ & 18:11:54.1 & 236.7 & $0.381282(1)$ & 27.5 & 16.848 & 0.422 \\
\hline V18 & $\mathrm{RRc}$ & $13: 12: 48.7$ & $18: 10: 12.9$ & 93.9 & $0.336054(1)$ & 22.2 & & \\
\hline V19 & RRc & $13: 13: 07.0$ & 18:09:26.1 & 173.2 & $0.391377(1)$ & 28.1 & 16.880 & 0.437 \\
\hline V20 & $\mathrm{RRc}$ & $13: 13: 09.0$ & $18: 04: 16.2$ & 404.7 & $0.384337(1)$ & 22.5 & 16.875 & 0.407 \\
\hline V23 & $\mathrm{RRc}$ & $13: 13: 02.3$ & $18: 08: 35.9$ & 137.9 & $0.365804(1)$ & 25.2 & 16.825 & 0.391 \\
\hline V24 & RRab & $13: 12: 47.2$ & 18:09:33.0 & 120.4 & $0.763198(2)$ & 20.3 & & \\
\hline V25 & RRab & $13: 13: 04.4$ & $18: 10: 37.2$ & 133.5 & $0.705162(1)$ & 16.7 & 16.779 & 0.555 \\
\hline V26 & $\mathrm{RRc}$ & $13: 12: 35.7$ & $18: 05: 20.5$ & 401.7 & $0.391106(1)$ & 24.1 & 16.845 & 0.395 \\
\hline V27 & RRab & $13: 12: 41.4$ & $18: 07: 23.9$ & 257.9 & $0.671071(1)$ & 14.7 & 16.856 & 0.550 \\
\hline V28 & RRab & $13: 12: 42.1$ & $18: 16: 37.9$ & 430.9 & $0.6327804(7)$ & 16.5 & 16.875 & 0.545 \\
\hline V29 & RRab & $13: 13: 04.3$ & 18:08:46.9 & 152.9 & $0.823243(4)$ & 26.6 & 16.782 & 0.588 \\
\hline V31 & RRab & $13: 12: 59.6$ & $18: 10: 04.6$ & 61.9 & $0.705665(1)$ & 15.2 & & \\
\hline V32 & $\mathrm{RRc}$ & $13: 12: 47.7$ & $18: 08: 35.9$ & 142.6 & $0.390623(2)$ & 20.4 & 16.712 & 0.416 \\
\hline V33 & RRab & $13: 12: 43.9$ & $18: 10: 13.2$ & 162.5 & $0.6245815(8)$ & 16.9 & .. & .. \\
\hline V34 & $\mathrm{RRc}$ & $13: 12: 45.7$ & $18: 06: 26.1$ & 262.0 & $0.289611(1)$ & 21.2 & 16.928 & 0.314 \\
\hline V35 & $\mathrm{RRc}$ & $13: 13: 02.4$ & $18: 12: 37.5$ & 179.8 & $0.372666(2)$ & 22.7 & 16.943 & 0.442 \\
\hline V36 & $\mathrm{RRc}$ & $13: 13: 03.3$ & $18: 15: 10.2$ & 321.8 & $0.373242(1)$ & 21.5 & 16.879 & 0.414 \\
\hline V37 & RRab & $13: 12: 52.3$ & $18: 11: 05.1$ & 69.6 & $0.717615(1)$ & 21.0 & .. & 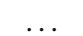 \\
\hline V38 & RRab & $13: 12: 57.1$ & $18: 07: 40.5$ & 151.8 & $0.705792(2)$ & 21.2 & 16.749 & 0.557 \\
\hline V40 & $\mathrm{RRc}$ & $13: 12: 55.9$ & $18: 11: 54.7$ & 105.3 & $0.3147939(9)$ & 19.6 & $\ldots$ & $\ldots$ \\
\hline V41 & RRab & $13: 12: 56.8$ & $18: 11: 09.3$ & 63.6 & $0.614438(1)$ & 19.2 & .. & $\cdots$ \\
\hline V42 & RRab & $13: 12: 50.6$ & $18: 10: 19.5$ & 67.4 & $0.713717(2)$ & 21.8 & & \\
\hline V43 & RRab & $13: 12: 53.1$ & $18: 10: 55.7$ & 55.5 & $0.712017(2)$ & 24.2 & .. & $\cdots$ \\
\hline V44 & $\mathrm{RRc}$ & $13: 12: 51.5$ & 18:09:58.2 & 54.6 & $0.375099(2)$ & 23.7 & .. & $\cdots$ \\
\hline V45 & RRab & $13: 12: 55.2$ & $18: 09: 27.4$ & 42.4 & $0.654950(2)$ & 16.2 & & \\
\hline V46 & RRab & $13: 12: 54.6$ & $18: 10: 36.2$ & 28.1 & $0.703655(3)$ & 22.0 & & \\
\hline V47 & $\mathrm{RRc}$ & $13: 12: 50.4$ & $18: 12: 24.6$ & 151.4 & $0.335377(1)$ & 21.1 & 16.791 & 0.372 \\
\hline V51 & $\mathrm{RRc}$ & $13: 12: 57.8$ & $18: 10: 50.7$ & 54.7 & $0.355203(2)$ & 20.2 & .. & $\cdots$ \\
\hline V54 & $\mathrm{RRc}$ & $13: 12: 54.4$ & $18: 10: 31.4$ & 25.0 & $0.315122(3)$ & 14.2 & $\ldots$ & \\
\hline V55 & $\mathrm{RRc}$ & $13: 12: 53.6$ & $18: 10: 39.2$ & 37.7 & $0.443386(2)$ & 26.4 & $\ldots$ & 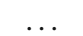 \\
\hline V56 & $\mathrm{RRc}$ & $13: 12: 53.7$ & $18: 09: 29.1$ & 46.3 & $0.328796(2)$ & 15.1 & $\ldots$ & 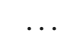 \\
\hline V57 & RRabB & $13: 12: 55.5$ & 18:09:58.0 & 12.1 & $0.568234(7)$ & 11.8 & .. & \\
\hline V58 & $\mathrm{RRc}$ & $13: 12: 55.6$ & 18:09:30.8 & 39.4 & $0.354954(3)$ & 17.2 & .. & \\
\hline V59 & $\mathrm{RRc}$ & $13: 12: 56.7$ & 18:09:20.4 & 53.4 & $0.303941(2)$ & 19.7 & $\ldots$ &. \\
\hline V60 & RRab & $13: 12: 57.0$ & 18:09:36.0 & 41.6 & $0.644755(2)$ & 18.4 & .. & \\
\hline V62 & $\mathrm{RRc}$ & $13: 12: 54.0$ & $18: 10: 30.1$ & 27.0 & $0.359891(4)$ & 13.8 & & \\
\hline V63 & $\mathrm{RRc}$ & $13: 12: 56.2$ & $18: 10: 02.8$ & 15.5 & $0.310476(4)$ & 14.3 & & .. \\
\hline V64 & $\mathrm{RRc}$ & $13: 12: 52.6$ & $18: 10: 12.5$ & 38.5 & $0.319529(1)$ & 19.2 & & \\
\hline V71* & $\mathrm{RRc}$ & $13: 12: 54.5$ & 18:09:54.3 & 18.8 & $0.304242(5)$ & 14.5 & & \\
\hline V72* & $\mathrm{RRc}$ & $13: 12: 55.8$ & 18:09:50.6 & 20.7 & $0.254155(3)$ & 13.5 & $\cdots$ & $\cdots$ \\
\hline
\end{tabular}

Notes:

The radial distance $d\left[{ }^{\prime \prime}\right]$ is measured from the nominal cluster center defined in Stetson's catalog of photometric standards (Stetson 2000; $\alpha=$ $13^{\mathrm{h}} 12^{\mathrm{m}} 55^{\mathrm{s}} .26 ; \delta=+18^{\circ} 10^{\prime} 09^{\prime} .8$ [2000.0]); errors corresponding to the last digits of the periods are shown in parentheses (see Sect. 3.1 for details on the computation of the errors); the signal-to-noise ratio (SNR) was calculated in the $[0.0,20.0] \mathrm{d}^{-1}$ frequency range; the $V$ and $V-I$ magnitude averages (i.e., the zero frequency constant in their Fourier decompositions) are given in those cases in which the flux-to-magnitude conversion was reliable (see Sect. 2 and Fig. 8); stars denote new detections, RRab, RRc and RRabB/RRcB stand for fundamental, first overtone and Blazhko variables, Fourier decompositions of the RR Lyrae stars with magnitude-transformed light curves in $V$ color are available online at CDS. 